\title{
Effect of resonance decays on hadron elliptic flows
}

\author{
V. Greco and C. M. Ko \\ Cyclotron Institute and Physics Department, Texas A\&M University, College Station, Texas 77843-3366, USA
}

(Received 9 February 2004; published 11 August 2004)

\begin{abstract}
Within the quark coalescence model, we study effects of resonance decays, and of the quark momentum distribution in hadrons, on the elliptic flows of stable hadrons. We find that, with the exception of rho-meson decays, the resonance decays could have a significant effect on pion elliptic flow. However, most secondary pions stem from the rho-meson decays, resulting in a reduced effect of resonance decays on their flow. Proton and kaon flows as well as the lambda flow are, however, not much affected by resonance decays. The distribution of quark momentum in hadrons also influences their elliptic flows, leading to a better agreement with experimental flow data when compared with the naive quark coalescence model, which only allows quarks with equal momentum to form a hadron.
\end{abstract}

DOI: 10.1103/PhysRevC.70.024901

PACS number(s): 25.75.Ld, 25.75.Nq

\section{INTRODUCTION}

A useful observable for understanding both the dynamics of heavy-ion collisions and the properties of produced hot and dense matter is the elliptic flow of hadrons, i.e., their momentum anisotropy in the transverse plane perpendicular to the beam directions. For heavy-ion collisions at the relativistic heavy ion collider (RHIC), it has been shown in studies based on hydrodynamical models [1-3] that hadron elliptic flows are sensitive to the equation of state of the quarkgluon plasma produced during the initial stage of the collision. In transport models [4-6], hadron elliptic flows are also shown to depend on the parton scattering cross sections in initial partonic matter. For hadrons with high transverse momentum, their elliptic flow can further provide information on the energy density of initial hot matter [7]. Experimentally, hadron elliptic flows in $\mathrm{Au}+\mathrm{Au}$ collisions at $\sqrt{s_{N N}}=130$ and $200 \mathrm{GeV}$ have been studied as functions of pseudo-rapidity [8], centrality $[9,10]$, and transverse momentum $[10,11]$. Furthermore, the elliptic flows of identified particles have been measured, and except for pions they essentially follow the quark number scaling, i.e., the dependence of hadron elliptic flows on hadron transverse momentum becomes similar if both are divided by the number of constituent quarks in a hadron, i.e., two for mesons and three for baryons. The scaling of hadron elliptic flows according to their constituent quark numbers has a simple explanation in the naive quark coalescence model [12], in which the meson elliptic flow at certain transverse momentum is given by twice the quark elliptic flow at half the meson transverse momentum, while that of baryons is given by three times the quark elliptic flow at one third of baryon transverse momentum. In more realistic quark coalescence models [13-17] that take into account the momentum distribution of quarks in hadrons, the scaled hadronic elliptic flows are, however, expected to be smaller than that of partons [18], and this may lead to a violation of the quark number scaling of hadron elliptic flows.

In this paper, we study how the elliptic flows of pions and other stable hadrons are affected by decays of resonances, such as $\rho \rightarrow 2 \pi, \omega \rightarrow 3 \pi, K^{*} \rightarrow K \pi$, and $\Delta \rightarrow N \pi$. For most hadrons, such as the proton, kaon, and lambda, including contributions from resonance decays do not affect much their elliptic flows. This is, however, different for pions as the elliptic flow of pions from the decays of most resonances, except the $\rho$ meson, are significantly different from that of directly produced pions. This effect is found to account for half of the observed deviation of pion elliptic flow from the quark number scaling. Taking into account the quark momentum distribution in hadrons, which makes it possible for quarks with different momenta to coalescence into hadrons, we find that the other half of the observed deviation can also be largely explained.

The paper is organized as follows. In Sec. II, we review observed approximate quark number scaling of the elliptic flows of identified hadrons and interpret it with the naive quark coalescence model. The effect of resonance decays on the elliptic flows of stable hadrons is then studied in Sec. III. In Sec. IV, we further investigate the effect on hadron elliptic flows due to quark momentum distribution inside hadrons. Finally, a summary is given in Sec. V.

\section{SCALING OF HADRON ELLIPTIC FLOWS}

In Fig. 1, we first show the experimental data from $\mathrm{Au}$ $+\mathrm{Au}$ collisions at $\sqrt{s_{N N}}=200 \mathrm{GeV}$ for the elliptic flows of pions (circles) and protons (squares) from the PHENIX Collaboration [19] as well as $K_{s}^{0}$ (diamonds) and lambdas (triangles) from the STAR Collaboration [20] scaled by their constituent quark numbers as functions of their transverse momentum per quark [21]. Except for pions, the scaled elliptic flows of other stable hadrons are indeed similar and can be described by the naive quark coalescence model [12] using the quark elliptic flow

$$
v_{2, q}\left(p_{T}\right)=v_{0} \tanh \left(\alpha p_{T}+\phi\right),
$$

with $v_{0}=0.078, \alpha=1.59$, and $\phi=-0.27$. In this model, the elliptic flows of mesons and baryons are related to that of quarks by $v_{2, \mathrm{M}}\left(p_{T}\right) \approx 2 v_{2, q}\left(p_{T} / 2\right)$ and $v_{2, \mathrm{~B}} \approx 3 v_{2, q}\left(p_{T} / 3\right)$, respectively, where small higher-order corrections are neglected [22]. The resulting scaled hadron elliptic flows are shown in Fig. 1 by the solid line, which is seen to give a 


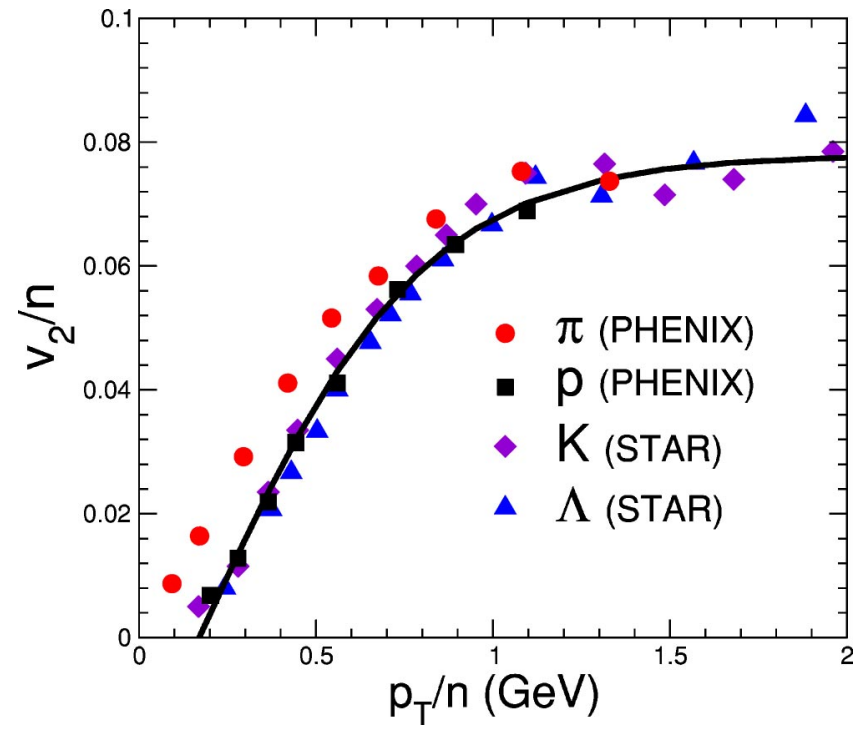

FIG. 1. (Color online) Experimental scaled elliptic flows as functions of scaled momentum for pions (circles) and protons (squares) from the PHENIX Collaboration [19] as well as kaons (diamonds) and lambdas (triangles) from the STAR Collaboration [20] in $\mathrm{Au}+\mathrm{Au}$ collisions at $\sqrt{s_{N N}}=200 \mathrm{GeV}$. The solid line given by Eq. (1) is a fit to the experimental data for hadrons other than pions.

good description of the experimental data for stable hadrons other than pions.

\section{EFFECT OF RESONANCE DECAYS}

A large fraction of stable hadrons such as pions, kaons, lambdas, and protons, detected in heavy ion collisions are from resonance decays. Although, in the coalescence model, the elliptic flow of both direct hadrons and resonances essentially follows the quark number scaling, hadrons from resonance decays may have elliptic flow that deviate from this scaling. To see the effect of resonance decays on the elliptic flow of stable hadrons, we use the coalescence model of Ref. [14] to generate their transverse momentum distributions. Specifically, soft partons with transverse momentum below $2 \mathrm{GeV} / c$, which are taken to have constituent quark masses, are assumed to have thermal spectra at temperature $T$ $=170 \mathrm{MeV}$ with a collective radial flow increasing linearly with radial distance up to a maximum flow velocity of $0.5 c$, while hard partons above $2 \mathrm{GeV} / c$ are given by the quenched minijet partons with power-like spectra. Furthermore, the volume of partonic matter at hadronization is taken to be $V=900 \mathrm{fm}^{3}$, leading to a parton density of about $1.15 \mathrm{fm}^{-3}$. The momentum spectra of hadrons are then obtained from the overlap between the parton distribution functions with the Wigner distribution functions of hadrons, which we evaluate using the Monte Carlo method of Ref. [14]. The hadron Wigner distribution functions are taken to have a momentum width of $\Delta_{p}=0.24 \mathrm{GeV} / c$ for mesons and $0.35 \mathrm{GeV} / c$ for baryons. These parameters have been shown in Ref. [14] to describe very well the measured transverse momentum spectra of pions, kaons, and protons.

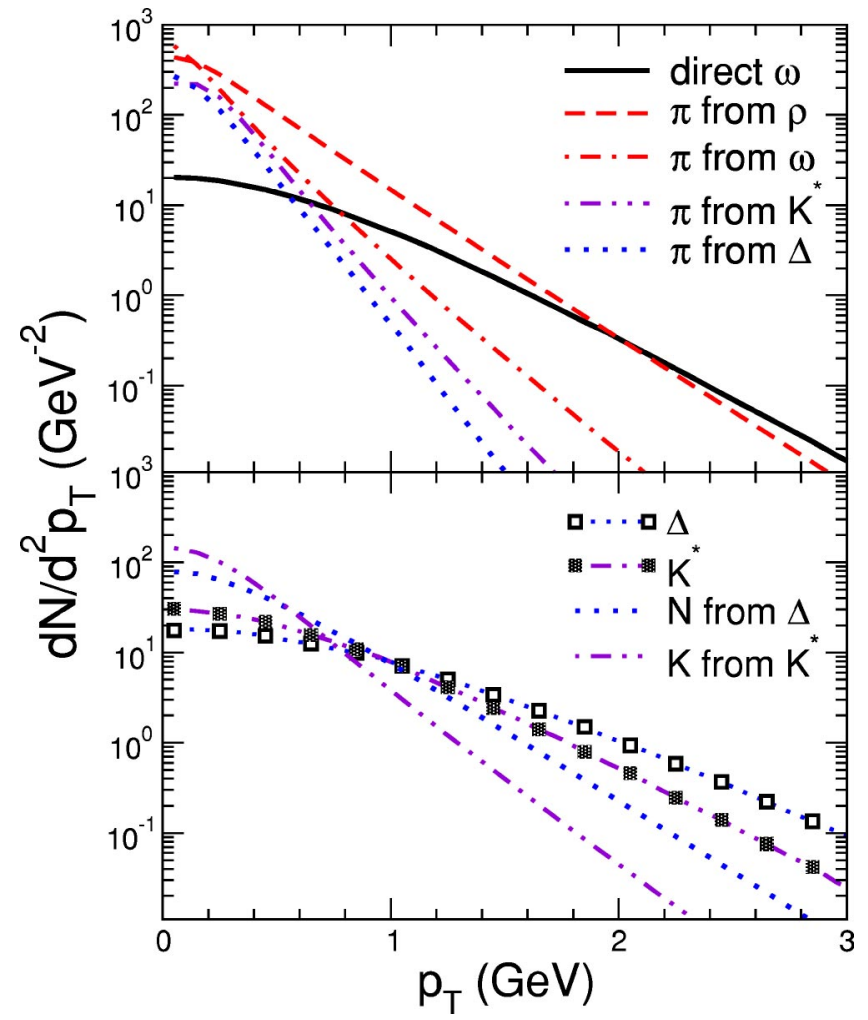

FIG. 2. (Color online) Transverse momentum spectra of hadrons. Upper panel: spectra of pions from decays of $\rho$ mesons (dashed line), $\omega$ mesons (dash-dotted line), $K^{*}$ (dash-dot-dotted line), and $\Delta$ (dotted line). The spectrum of $\omega$ mesons is shown by the solid line. Lower panel: Spectra of $\Delta$ (dotted line with open squares) and $K^{*}$ (dash-dot-dotted line with shaded squares) together with those of nucleons (dotted line) and $K$ (dash-dot-dotted line) from their decays.

\section{A. Transverse momentum spectra}

We first show in Fig. 2 the transverse momentum spectra of various hadrons obtained from the quark coalescence model of Refs. [13,14]. In the upper panel, the spectrum of $\omega$ mesons is given by the solid line, and that of pions from $\omega$ decays is given by the dash-dotted line and is seen to be steeper than the original $\omega$ meson spectrum. The same is true for the transverse momentum spectra of pions from decays of $\rho$ meson (dashed line), $K^{*}$ (dash-dot-dotted line), and $\Delta$ (dotted line), i.e., they are steeper than the transverse momentum spectra of original resonances. These pions, except from $\rho$ meson decays, also have steeper transverse momentum spectra than that of pions from $\omega$ decays. We note that in the coalescence model more pions are produced from $\rho$ meson decays than from the decays of other resonances and from direct recombination of quarks and antiquarks.

In the lower panel of Fig. 2, we show the initial transverse momentum spectra of $\Delta$ (dotted line with open squares) and $K^{*}$ (dash-dot-dotted line with shaded squares) as well as those of nucleons (dotted line) and $K$ (dash-dot-dotted line) from their decays. The differences between the slope parameters of nucleon and $K$ transverse momentum spectra and those of the parent $\Delta$ and $K^{*}$ are smaller than those between the slope parameters of the transverse momentum spectra of 
pions and their parent resonances. Our results on the transverse momentum spectra of resonances and their decay products are consistent with those using the thermal model [23], although there are differences in detail due to different hadron abundances and the inclusion of flow effects in our study.

\section{B. Elliptic flows}

To study the effect of resonance decays on the elliptic flow of stable hadrons, we assume that all resonances produced via coalescence have the scaled elliptic flow given by Eq. (1), as in the naive quark coalescence model. Since a decay particle at given transverse momentum arises from a resonance at higher momentum with identical elliptic flow, decay products are expected to have a larger elliptic flow than directly produced particles. For low-momentum particles, however, the decay process, which is isotropic in the rest frame of the resonance, reduces the momentum anisotropy (and thus the elliptic flow). At very low momentum, this effect can be large enough to render the elliptic flow of particles from resonance decays even smaller than that of directly produced ones, as first pointed out in Ref. [24]. On the other hand, particles at large momentum are essentially aligned with the parent resonances, and consequently tend to preserve the elliptic flow of the parent resonances. These features are indeed seen in Fig. 3, where the scaled elliptic flow of stable hadrons from resonance decays is shown.

In the upper panel of Fig. 3, we show the scaled elliptic flows of pions from decays of various resonances. For pions from $\rho$ meson decay, the scaled elliptic flow, given by the dashed line, differs very little from that of directly produced pions, which in the naive quark coalescence model follows the quark number scaling given by the solid line. The slight enhancement of the pion elliptic flow at larger transverse momenta due to rho meson decays has also been seen previously in studies based on the thermal model [25]. The dashdotted line is the scaled pion elliptic flow from the decay of $\omega$ mesons. It has a larger value than that of directly produced pions as the average transverse momentum of these pions is even smaller than that of pions from $\rho$ meson decays as shown in Fig. 2. Similarly, the elliptic flow of pions from $K^{*}$ decays (dash-dot-dotted line) is large and comparable to that of pions from $\omega$ decays. For pions from $\Delta$ decays (dotted line), their elliptic flow is even larger than that for pions from $\omega$ and $K^{*}$ decays. Therefore, pions from resonance decays can lead to a deviation of the final pion elliptic flow from the quark number scaling. As shown in the lower panel, the elliptic flow of pions including those from resonance decays (dash-dotted line) is indeed closer to the experimental data (solid circles) than that of directly produced pions (solid line) expected from the naive quark coalescence model. The effect of resonance decays on the pion elliptic flow is thus not small and can roughly account for half of the observed deviation of pion elliptic flow from the quark number scaling.

In the middle panel of Fig. 3, we show the elliptic flows of $K$ (dash-dot-dotted line) and nucleons (dotted line) from decays of $K^{*}$ and $\Delta$, and they are seen to differ only slightly from those of directly produced ones, which again follow more or less the quark number scaling given by the solid

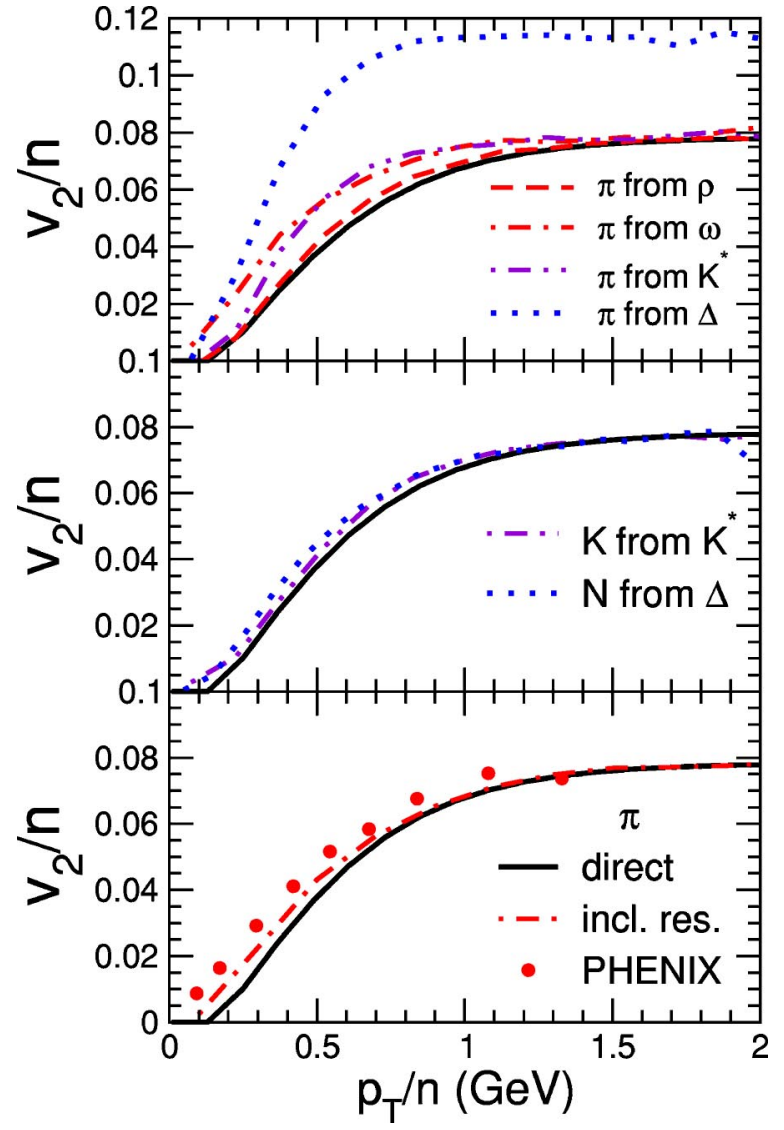

FIG. 3. (Color online) Scaled hadron elliptic flows as functions of scaled momentum. Upper panel: pions from decays of $\rho$ mesons (dashed line), $\omega$ (dash-dotted line), $K^{*}$ (dash-dot-dotted line), and $\Delta$ (dotted line). Middle panel: $K$ from $K^{*}$ decays (dash-dot-dotted line) and protons from $\Delta$ decays (dotted line). Lower panel: Pions including resonance decays (dash-dotted line) and from experimental data (solid circles). The solid line in all panels represents the scaled elliptic flow [Eq. (1)] obtained from fitting the experimental data for stable hadrons other than pions.

line. Resonance decays thus do not destroy the observed approximate scaling of proton, kaon, and lambda elliptic flows according to their constituent quark number. The difference between the elliptic flows of hadrons and their decay products has also been studied for charmed mesons [26]. It was found that the elliptic flow of electrons from charmed meson decays was also slightly larger than that of charmed mesons.

The above results are obtained without taking into account the width of resonances. Because of their finite widths, resonances are produced with masses given by the BreitWigner distribution. Since the momenta of pions from decays of resonances with masses below (above) their peak mass are smaller (larger) than the momenta of pions from decays of resonances with peak mass, the elliptic flows of these pions are larger (smaller). As a result, the increased elliptic flow of pions from resonances with masses below their peak value is canceled by the decreased elliptic flow of pions from resonances with masses above their peak values. Including the effect of resonance width is thus not expected to affect the above results. We have checked this for the $\rho$ meson, which dominates pion production in the coalescence 
model and has a width of $150 \mathrm{MeV}$, and indeed we have found that the resulting pion elliptic flow is essentially the same as the one without including the rho meson width. This remains so even if we increase the $\rho$ meson width to $300 \mathrm{MeV}$ due to possible collision broadening in dense medium [27].

Since a lower rho meson mass leads to a larger pion elliptic flow, it is interesting to note that the remaining deviation of scaled pion elliptic flow from the quark number scaling could be explained if the rho meson mass is reduced from $770 \mathrm{MeV} / c^{2}$ to $500 \mathrm{MeV} / c^{2}$. A dropping rho meson mass in dense medium has been suggested to relate to the partial restoration of chiral symmetry [28]. It has also been shown to give a possible explanation $[29,30]$ for the enhanced production of low mass dileptons observed in heavy ion collisions at the SPS [31]. More recently, the $\rho$ meson mass extracted from the two-pion invariant mass spectrum in heavy ion collisions at RHIC was found about $70 \mathrm{MeV} / \mathrm{c}^{2}$ less than its value in free space [32]. However, before one can draw such an conclusion, it is important to investigate other conventional effects that have been neglected in the naive quark coalescence model, and this is discussed in the next section.

\section{EFFECT OF QUARK MOMENTUM DISTRIBUTION IN HADRONS}

The scaling of hadron elliptic flows with their constituent quark number only holds in the naive quark coalescence model that requires mesons at transverse momentum $p_{T}$ to be formed from partons at momentum $p_{T} / 2$ and baryons from quarks at momentum $p_{T} / 3$. In more realistic coalescence models, hadron production is expressed in terms of the overlap of parton distributions with the Wigner functions of formed hadrons, so quarks with different momenta can coalescence into hadrons [14]. As shown in Ref. [18], in which only collinear quarks can form hadrons, the effect due to the momentum distribution of quarks in hadrons can lead to a breaking of quark number scaling if the quark elliptic flow does not depend linearly on the transverse momentum. To study this effect quantitatively, we again use the Monte Carlo method of Ref. [14] to evaluate the coalescence integral so that noncollinear quarks can also form hadrons. To obtain scaled hadron elliptic flows that are comparable to the measured ones, we further take quarks to have an elliptic flow similar to Eq. (1) but with $v_{0}=0.089, \alpha=1.89$, and $\phi=-0.22$. This gives a larger quark elliptic flow, shown by the solid line in Fig. 4, than the quark elliptic flow in Fig. 1. The resulting scaled meson (dashed line) and baryon (dotted line) elliptic flows are shown in Fig. 4, and they are smaller than the quark elliptic flow. This effect is larger for baryons than for mesons, leading thus to a scaled elliptic flow that is smaller for baryons than for mesons.

Including also the contribution from decays of resonances, the resulting scaled pion (dashed line) and proton (dotted line) elliptic flows are shown in Fig. 5 together with the experimental data [19] for pions (circles) and protons (squares). The observed difference in the scaled pion and proton elliptic flows can be largely explained as already

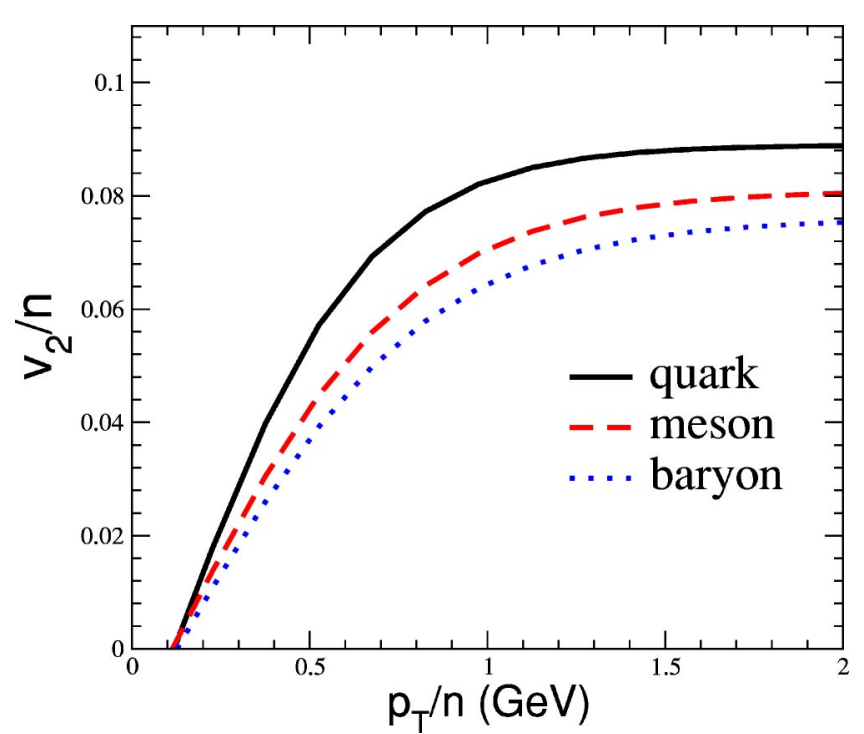

FIG. 4. (Color online) Scaled elliptic flows of quarks (solid line), meson (dashed line) and baryons (dotted line) in a realistic quark coalescence model.

shown in Ref. [14]. It was also shown in Ref. [14] that the more realistic coalescence model could describe the measured elliptic flow of kaons and lambdas if the underlying elliptic flow of strange quarks is taken to be slightly different from that of light quarks. Therefore, a combination of the effects due to the quark momentum distribution in hadrons and resonance decays in the coalescence model can lead to the observed quark number scaling of hadron elliptic flows as well as the violation of the pion elliptic flow from this scaling behavior.

The measured hadron elliptic flows including that of pions for transverse momentum $p_{T} \leqslant 1.5 \mathrm{~A} \mathrm{GeV}$ have also been

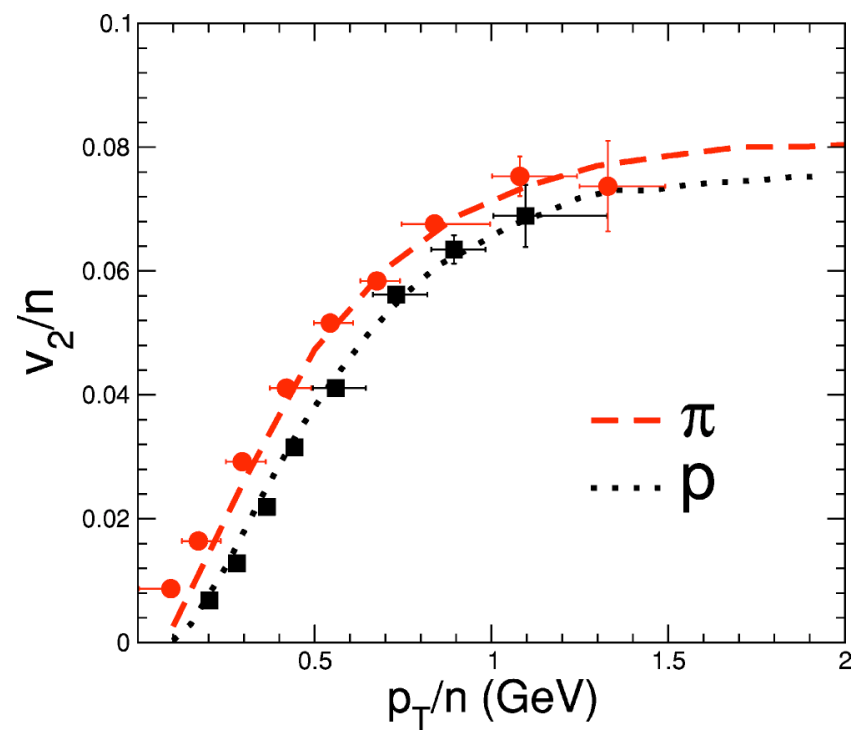

FIG. 5. (Color online) Scaled pion (dashed line) and proton (dotted line) elliptic flows in the coalescence model that includes effects due to both resonance decays and the quark momentum distribution in hadrons. Experimental data are shown by circles for pions and squares for protons [19]. 
explained by the hydrodynamical model [3], which predicts that hadron elliptic flows are affected by their masses with heavier ones having larger elliptic flows. As a result, the observed violation of scaled pion elliptic flow from the quark number scaling can be attributed to its small mass. Since the phi meson mass is similar to that of proton but is a meson, its elliptic flow is similar to that of proton in the hydrodynamical model and is thus expected to deviate also from the quark number scaling. This is in contrast to the prediction of the quark coalescence model that the phi meson elliptic flow should satisfy the quark number scaling as well [14]. Measurement of the phi meson elliptic flow in relativistic heavy ion collisions is thus important for testing the different predictions from these two models [33].

In the above study, we have neglected the effect of rescattering on hadron elliptic flows. This is justified as studies based on transport models have shown that elliptic flows in heavy ion collisions are generated during the initial stage of collisions when both spatial and pressure anisotropies are appreciable, and their values remain essentially unchanged during hadronic evolution [6,34]. Only for resonances with transverse momentum $p_{T} / n \simeq 1.5 \mathrm{GeV}$, where $n$ is the number of constituent quarks in a resonance, their elliptic flows may be affected by hadronic rescattering effect [35].

\section{SUMMARY}

In summary, we have studied the effect of resonance decays on the elliptic flows of stable hadrons, particularly that of pions. For heavier hadrons such as $K$ and protons, their elliptic flows are not much affected by decays of $K^{*}$ and $\Delta$. This is different for pions as the elliptic flows of pions from resonance decays, except the $\rho$ meson, are larger than that of directly produced pions. Since $\rho$ meson decays are more important than other resonances in contributing to the final yield of pions, the final pion elliptic flow is, however, only moderately affected by resonance decays, leading to a deviation of the pion elliptic flow from the quark number scaling that is about half the observed one. Including the effect of quark momentum distribution in hadrons not only reduces the hadron elliptic flows but also leads to additional deviation of the scaled pion elliptic flow from that of protons. The quark coalescence model that takes into account both effects of resonance decays and quark momentum distribution in hadrons thus can give a good description of not only the hadron transverse momentum spectra but also their elliptic flows. The agreement between theory and experiments at low momenta may, however, be fortuitous as the coalescence model, which ignores energy conservation, is likely to be too crude for hadrons with low momenta. To improve the model, one needs to take into account the effect of other partons in the system in order to balance the energy mismatch during quark coalescence, which is beyond the scope of present study.

Note added: After submission of this paper, we were aware of a paper by X. Dong, S. Esumi, P. Sorensen, and N. $\mathrm{Xu}$ [36] which also reaches a similar conclusion that resonance decays affect appreciably the pion elliptic flow.

\section{ACKNOWLEDGMENTS}

We are grateful to Lie-Wen Chen, Ralf Rapp, and $\mathrm{Nu} \mathrm{Xu}$ for helpful discussions. This paper was based on work supported in part by the U.S. National Science Foundation under Grant No. PHY-0098805 and the Welch Foundation under Grant No. A-1358. V.G. was also supported by the National Institute of Nuclear Physics (INFN) in Italy.
[1] D. Teaney, J. Laureant, and E. V. Shuryak, Phys. Rev. Lett. 86, 4783 (2001).

[2] P. F. Kolb, P. Huovinen, U. Heinz, and H. Heiselberg, Phys. Lett. B 500, 232 (2001).

[3] P. Huovinen, P. F. Kolb, U. Heinz, P. V. Ruuskanen, and S. Voloshin, Phys. Lett. B 503, 58 (2001).

[4] B. Zhang, M. Gyulassy, and C. M. Ko, Phys. Lett. B 455, 45 (1999).

[5] D. Molnar and M. Gyulassy, Nucl. Phys. A A698, 379 (2002).

[6] Z. W. Lin and C. M. Ko, Phys. Rev. C 65, 034904 (2002).

[7] M. Gyulassy, I. Vitev, and X. N. Wang, Phys. Rev. Lett. 86, 2537 (2001).

[8] S. Manly (for the PHOBOS Collaboration), Nucl. Phys. A A715, 611 (2003).

[9] K. H. Ackermann et al. (STAR Collaboration), Phys. Rev. Lett. 86, 402 (2001).

[10] S. Esumi (for the PHENIX Collaboration), Nucl. Phys. A A715, 599 (2003).

[11] C. Adler et al. (STAR Collaboration), Phys. Rev. Lett. 90, 032301 (2003).

[12] D. Molnar and S. A. Voloshin, Phys. Rev. Lett. 91, 092301 (2003).
[13] V. Greco, C. M. Ko, and P. Lévai, Phys. Rev. Lett. 90, 202302 (2003).

[14] V. Greco, C. M. Ko, and P. Lévai, Phys. Rev. C 68, 034904 (2003).

[15] R. J. Fries, B. Müller, C. Nonaka, and S. A. Bass, Phys. Rev. Lett. 90, 202303 (2003).

[16] R. J. Fries, B. Müller, C. Nonaka, and S. A. Bass, Phys. Rev. C 68, 044902 (2003).

[17] R. C. Hwa and C. B. Yang, Phys. Rev. C 67, 034902 (2003); 67, 064902 (2003).

[18] Z. W. Lin and D. Molnar, Phys. Rev. C 68044901 (2003).

[19] S. S. Adler et al. (PHENIX Collaboration), Phys. Rev. Lett. 91, 182301 (2003).

[20] P. Sorensen, J. Phys. G 30, S217 (2004); P. Sorensen, Ph.D Thesis, nucl-ex/0309003.

[21] Here and elsewhere in the paper, we use $\pi$ for both $\pi^{+}$and $\pi^{-}$, $p$ for both $p$ and $\bar{p}$. The same is for other particles, i.e., they denote both particles and their antiparticles.

[22] P. Kolb, L. W. Chen, V. Greco, and C. M. Ko, Phys. Rev. C 69, 051901(R) (2004).

[23] J. Sollfrank, P. Koch, and U. Heinz, Phys. Lett. B 252, 256 (1990). 
[24] T. Hirano, Phys. Rev. Lett. 86, 2754 (2001).

[25] W. Broniowski, A. Baran, W. Florkowski, AIP Conf. Proc. 660: 185-195 (2003); nucl-th/0212053.

[26] V. Greco, C. M. Ko, and R. Rapp, nucl-th/0312100.

[27] R. Rapp and J. Wambach, Adv. Nucl. Phys. 25, 1 (2000).

[28] G. E. Brown and M. Rho, Phys. Rev. Lett. 66, 2720 (1991); Phys. Rep. 363, 85 (2002).

[29] G. Q. Li, C. M. Ko, and G. E. Brown, Phys. Rev. Lett. 75, 4007 (1995); Nucl. Phys. A A606, 568 (1996); G. Q. Li, C. M. Ko, G. E. Brown, and H. Sorge, ibid. A611, 539 (1996); C. M. Ko, G. Q. Li, G. E. Brown, and H. Sorge, ibid. A610, 342c (1996).
[30] W. Cassing, W. Ehehalt, and C. M. Ko, Phys. Lett. B 363, 35 (1995)

[31] G. Agakichev et al., Phys. Rev. Lett. 75, 1272 (1995).

[32] P. Fachini (for the STAR Collaboration), Nucl. Phys. A A715, 462 (2003).

[33] C. Nonaka, R. J. Fries, S. A. Bass, Phys. Lett. B 583, 73 (2003).

[34] L. W. Chen, C. M. Ko, and Z. W. Lin, Phys. Rev. C 69, 031901 (2004).

[35] C. Nonaka, M. Asakawa, S. A. Bass, R. J. Fries, and B. Mueller, Phys. Rev. C 69, 031902 (2004).

[36] X. Dong, S. Esumi, P. Sorensen, and N. Xu, nucl-th/0403030. 